\title{
EXPERIMENTO ACADÊMICO: APLICAÇÃO DO MÉTODO DE DIFERENÇAS FINITAS PARA A ANÁLISE DO RESFRIAMENTO DE UMA BETERRABA COZIDA
}

\section{ACADEMIC EXPERIMENT: APPLICATION OF THE FINITE DIFFERENCE METHOD FOR THE ANALYSIS OF THE COOLING PROCESS OF A COOKED BEET}

\author{
A. L. S. MAGALHÃES ${ }^{1}$, S. V. BARBOSA ${ }^{1}$, K.V. S. OLIVEIRA ${ }^{1}$, A. P. S. ALVES ${ }^{1}$, G. A. S. ABREU ${ }^{1}$, M. \\ C. SILVA ${ }^{1}$, A. M. T. ERVILHA ${ }^{1}$ e S. F. ANTONIAZZI ${ }^{1}$, A. M. O. SIQUEIRA ${ }^{1}$
}

${ }^{1}$ Universidade Federal de Viçosa, Departamento de Química, Viçosa, Minas Gerais, Brasil

\section{A R T I C L E I N F O}

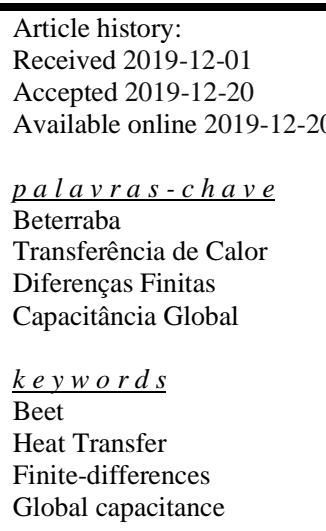

\section{RESUMO}

Diante da crescente preocupação com a segurança alimentar e seus respectivos parâmetros, estabelecidos pelos órgãos responsáveis, é importante que se saiba a respeito do tempo máximo de exposição de um alimento ao ar atmosférico de forma a evitar possíveis contaminações microbiológicas. A determinação do perfil de temperaturas nos alimentos e a forma como este varia ao longo do tempo permitem um maior controle sobre esse fator. Dessa forma, o presente estudo tem o objetivo de averiguar, por meio dos métodos numéricos de diferenças finitas e capacitância global, o processo de resfriamento de uma beterraba cozida, estabelecendo o tempo após o qual o alimento atingiria a temperatura de $60^{\circ} \mathrm{C}$ e estaria, assim, suscetível a contaminação. Após o cozimento do legume em questão, foram mensuradas as temperaturas em pontos de suas faces para intervalos de tempo preestabelecidos. Os resultados obtidos mostraram que, antes mesmo das aferições em tempos futuros, já existiam pontos na superfície às temperaturas de 52,9 e 56,6 ${ }^{\circ} \mathrm{C}$, indicando que o método utilizado não foi satisfatório para a análise inicialmente proposta.

\section{ABSTRACT}

Given the increasing concern about food safety and its parameters, set by government agencies, it is important to know about the maximum exposure time of food in general to atmospheric air, in order to avoid possible microbiological contamination. Defining the food temperature profile and its variation over time, allows greater control over this factor. Therefore, the present article aims to determine, through finite-difference and Global capacitance equations, the cooling process of a cooked beet, establishing the time after which the beet would reach the temperature of $60^{\circ} \mathrm{C}$, and be susceptible to contamination. After cooking the vegetable, surface temperatures were measured for predefined periods of time. The results revealed that, even before the future measurements, two of the surface points were already at the temperatures of 52.9 and $56.6^{\circ} \mathrm{C}$, showing that the used method was not satisfying for the initially proposed analysis. 


\section{INTRODUÇÃO}

O setor de alimentos no Brasil movimenta bilhões de reais anualmente. Fatores geográficos e climáticos permitem uma enorme variabilidade de alimentos na mesa dos brasileiros. De acordo com ABIA - Associação Brasileira da Indústria de Alimentos, o setor de alimentos registrou um faturamento de R\$656 bilhões no ano de 2018, incluindo carnes, legumes, frutas, entre outros. Os legumes, em particular, possuem uma grande participação no comércio e consumo nacional, de forma que condições de higienização e sanitização devem ser regularmente empregadas nos pontos de venda e de consumo (ABIA, 2019).

Todos os envolvidos na cadeia de produção são responsáveis por manter a segurança e qualidade dos alimentos. Produtores rurais, por exemplo, devem garantir a qualidade de sua produção, respeitando as normas e leis vigentes estabelecidas pelos órgãos de fiscalização. A indústria e o comércio devem apresentar condições de higiene estabelecidas por lei para armazenar e comercializar os produtos, e consumidores finais devem evitar contaminações durante o preparo dos alimentos, visto que, de acordo com a ANVISA Agência Nacional de Vigilância Sanitária (2005) com base nos dados epidemiológicos do Ministério da Saúde, mais de $45 \%$ das doenças transmitidas por alimentos são contraídas nas residências.

Cozinhar legumes é uma prática convencional em casas e restaurantes. O cozimento confere ao alimento uma textura apropriada, aroma agradável e melhor digestibilidade ao consumidor. Além disso, o ato de transferir calor para os alimentos até alcançar temperaturas superiores a $60{ }^{\circ} \mathrm{C}$, prolonga a estabilidade dos alimentos. Isso ocorre devido à morte dos microrganismos, por uma provável mudança irreversível no protoplasma a ponto de não mais funcionar, por desnaturação e coagulação dos microrganismos, ou, até mesmo, por destruição de enzimas essenciais (BALL e OLSON, 1957).

Durante o cozimento, fatores como dimensões dos alimentos, formato e tempo submerso na água, geram variações internas e externas de temperatura. Existe, portanto, a possibilidade de avaliar o perfil de temperaturas do alimento ao retirá-lo do cozimento e a sua respectiva variação ao longo do tempo. A análise dessa variação, por sua vez, permite inferir o quanto os alimentos podem ficar expostos à atmosfera de modo a evitar a contaminação microbiológica que pode ocorrer dependendo do tempo de exposição e da faixa de temperatura atingida.

Utilizando técnicas de cálculo, física, balanço de energia e transferência de calor, é possível avaliar o tempo em que o alimento alcançará temperaturas inferiores às de segurança. Diversos estudos utilizam técnicas analíticas e algébricas para avaliar o tempo de resfriamento frente as trocas de calor, principalmente por convecção, de frutas e vegetais, empregando diferentes restrições e métodos computacionais avançados (AMORIM, 2016; AMÊNDOLA e TUREL, 2005; TERUEL, et al., 2001).
No que diz respeito à confiabilidade do método em questão, os cálculos do coeficiente de transferência de calor por convecção $(h)$ e medições experimentais da temperatura na superfície dos alimentos são as causas mais comuns das elevadas incertezas usualmente obtidas, de modo que é necessário aprimorar essa etapa nos estudos em desenvolvimento (ANDRADE et al., 2017).

Assim sendo, o objetivo do presente trabalho é utilizar a técnica numérica de diferenças finitas aplicada à transferência de calor para avaliar o processo de resfriamento de uma beterraba cozida ao longo do tempo. Dessa forma, torna-se possível quantificar o tempo máximo de exposição do alimento ao ar após o cozimento, evitando que se atinja a temperatura mínima estabelecida pelas normas de segurança alimentar.

\section{CONCEITOS FUNDAMENTAIS}

Nessa seção são apresentados conceitos fundamentais para a compreensão dos resultados a serem exibidos posteriormente. $\mathrm{O}$ tópico 2.1 diz respeito à transferência de calor de forma geral, e seus respectivos modos. O tópico 2.2, por sua vez, trata do processo utilizado para determinar o coeficiente de transferência de calor por convecção. Por fim, nos tópicos 2.3 e 2.4, apresentam-se os métodos numéricos empregados no tratamento de dados, sendo eles diferenças finitas e capacitância global, respectivamente.

\subsection{Transferência de calor}

A transferência de calor ocorre entre dois corpos que se encontram em temperaturas diferentes e pode acontecer por três formas distintas: condução, convecção e irradiação. De forma geral, quando há transferência de calor esses três processos ocorrem simultaneamente, mas em certos casos há a predominância de algumas das formas, podendo desprezar-se as outras.

Para expressar os fenômenos de transferência de calor, é necessário conhecer o conceito de "fluxo de calor". Esse fluxo é representado pelo símbolo q" e é definido como a quantidade de calor transferido por unidade de área perpendicular à direção da transferência (DA ROSA et al., 2016; GARCIA et al., 2017).

O fenômeno da condução é visto como o transporte de energia entre partículas devido à existência de um gradiente de temperatura (RODRIGUES, 2011). Quando as partículas de um material se chocam umas com as outras, ocorre transferência de energia das partículas mais energéticas para as menos energéticas (INCROPERA et al., 2008). O fluxo térmico em um meio é determinado pela Lei de Fourier:

$$
q_{\text {cond }}=-k\left(i \frac{\partial T}{\partial x}+j \frac{\partial T}{\partial y}+k \frac{\partial T}{\partial z}\right)
$$

em que q" cond é o fluxo de calor por condução, $\left[\mathrm{Wm}^{-2}\right]$, k é a condutividade térmica do material, $\left[\mathrm{Wm}^{-1} \mathrm{~K}^{-1}\right], \mathrm{T}$ é a temperatura, $[\mathrm{K}]$, e $\mathrm{x}, \mathrm{y}$ e $\mathrm{z}$ são as coordenadas espaciais cartesianas, [m]. 
É possível, ainda, realizar um balanço de energia para um volume no qual está havendo transferência de calor por condução, obtendo-se a equação da condução do calor, que também leva em consideração parcelas de acúmulo e de geração

$$
\frac{\partial^{2} T}{\partial x^{2}}+\frac{\partial^{2} T}{\partial y^{2}}+\frac{\partial^{2} T}{\partial z}+\frac{\dot{q}}{k}=\frac{1}{\alpha} \frac{\partial T}{\partial t}
$$

onde q̇ é a taxa de geração de energia por unidade de volume, $\left[\mathrm{Wm}^{-3}\right], \mathrm{t}$ representa o tempo, [s], e $\alpha$ é a difusividade térmica do material, $\left[\mathrm{m}^{2} \mathrm{~s}^{-1}\right]$, a qual é definida como a razão entre a condutividade térmica e o produto entre a massa específica e a capacidade calorífica, $\left[\mathrm{Jkg}^{-1} \mathrm{~K}^{-1}\right]$ :

$$
\alpha=\frac{k}{\rho c_{p}}
$$

Já a convecção é uma característica dos fluidos e está associada tanto ao movimento molecular aleatório quanto ao movimento macroscópico do fluido. Essa forma de transferência de calor ocorre quando uma superfície de certa temperatura entra em contato com um fluido em movimento de temperatura diferente (INCROPERA et al., 2008). O fluxo de calor por convecção (q" conv) é descrito pelo produto entre o coeficiente de transferência de calor por convecção $(\mathrm{h}),\left[\mathrm{Wm}^{-2} \mathrm{~K}^{-1}\right]$, e a diferença entre as temperaturas da superfície em questão $\left(\mathrm{T}_{\mathrm{s}}\right)$ e do fluido circundante $\left(\mathrm{T}_{\infty}\right)$, ou seja, (GARCIA et al., 2017):

$$
q^{\prime \prime}{ }_{\text {conv }}=h\left(T_{s}-T_{\infty}\right)
$$

O coeficiente de transferência de calor por convecção depende da geometria da superfície, da natureza do escoamento do fluido, entre outras propriedades termodinâmicas e de transporte do fluido (INCROPERA et al., 2008).

Por último, na irradiação o calor é transmitido por meio de ondas eletromagnéticas, não necessitando de um meio material para ocorrer (DA ROSA et al., 2016). A quantidade de energia irradiada é função da temperatura do material e da direção na qual a radiação é emitida. $O$ corpo que emite a máxima radiação possível é chamado de radiador ideal. O fluxo de transferência de calor por radiação (q", rad) é descrito pela lei de Stefan-Boltzmann, dada por (GARCIA et al., 2017):

$$
q^{\prime \prime}{ }_{\text {rad }}=\varepsilon \sigma\left(T_{S}^{4}-T_{v i z}^{4}\right)
$$

em que $\varepsilon$ é a emissividade, [ ], o qual fornece a eficiência com que uma superfície emite energia em relação ao radiador ideal,

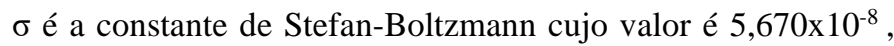
$\left[\mathrm{Wm}^{-2} \mathrm{~K}^{-4}\right], \mathrm{T}_{\mathrm{S}}$ é a temperatura da superfície do objeto, $[\mathrm{K}]$, e $\mathrm{T}_{\text {viz }}$ é a temperatura da superfície vizinha que envolve o sólido, de energia. Considerando a condutividade térmica do material constante em todo o volume, chega-se na seguinte relação da equação do calor:

[K], considerada muito maior que o próprio sólido (INCROPERA et al., 2008).

\subsection{Determinação do coeficiente de transferência de calor por convecção}

Para a análise dos dados pelo método de diferenças finitas, faz-se necessária a utilização de três números adimensionais para o cálculo do coeficiente de transferência de calor por convecção do ar. São eles: número de Nusselt, Rayleigh e Prandtl.

O processo de transferência de calor envolvendo fluidos pode se dar por condução, quando o fluido está estático, ou por convecção, quando há movimento desse fluido. $\mathrm{O}$ número de Nusselt $(\mathrm{Nu})$ é responsável por adimensionalizar o coeficiente de transferência de calor, aglutinando os coeficientes de transferência de calor por condução e convecção em um só parâmetro. Esse número adimensional é definido pela seguinte equação:

$$
N u=\frac{h L}{k_{a r}}
$$

onde $L$ é o comprimento característico definido como a razão entre a área superficial, $\left[\mathrm{m}^{2}\right]$, e o perímetro da placa, $[\mathrm{m}]$.

O número de Rayleigh $(R a)$, por sua vez, está associado à condução por convecção natural, ou seja, esse número adimensional define se a transferência de calor é realizada essencialmente por condução ou por convecção.

$$
R a=\frac{\rho_{a r} g\left(T_{s}-T_{\infty}\right) L_{c}{ }^{3}}{T_{f} \mu_{a r} \alpha_{a r}}
$$

onde $\rho_{a r}$ é a massa específica do ar, $\left[\mathrm{kg} \mathrm{m}^{-3}\right], g$ é a aceleração da gravidade, $\left[\mathrm{m} \mathrm{s}^{-2}\right], T_{s}$ é a temperatura da superfície do sólido, calculada a partir da média das temperaturas dos pontos tomados, $[\mathrm{K}], T_{\infty}$ é a temperatura ambiente, $[\mathrm{K}], \mu_{a r}$ é a viscosidade dinâmica do ar, $\left[\mathrm{N} \mathrm{s} \mathrm{m}^{-2}\right]$ e $T_{f}$ é a temperatura média da camada limite, $[\mathrm{K}]$ :

$$
T_{f}=\frac{T_{s}+T_{\infty}}{2}
$$

Já o número de Prandtl, fornece a relação entre as espessuras das camadas limite térmica e hidrodinâmica através 
da razão entre a viscosidade cinemática do fluido e sua difusividade térmica (ÇENGEL e GHAJAR, 2012).

$$
\operatorname{Pr}=\frac{v_{a r}}{\alpha_{a r}}=\frac{C_{p_{a r}} \mu_{a r}}{k_{a r}}
$$

\subsection{Métodos de diferenças finitas}

Os métodos analíticos possibilitam o cálculo da solução exata das incógnitas de um problema de transferência de calor. Todavia, estas soluções são conhecidas apenas para alguns casos específicos, os quais se distanciam das aplicações práticas do cotidiano. Assim, faz-se necessário utilizar procedimentos aproximados, que podem ser aplicados de forma geral, desde que estejam condizentes com precisão aceitável estabelecida para problemas de engenharia. Os métodos de diferenças finitas consistem em exemplos desses procedimentos numéricos aproximados (SILVA et al., 2007).

$\mathrm{O}$ princípio fundamental destes métodos consiste em transformar a resolução de uma equação diferencial em um sistema de equações algébricas ao substituir as derivadas por diferenças (RUGGIERO; LOPES, 1996).

Considera-se o sistema bidimensional como representado na Figura 1, onde cada ponto $\left(x_{i}, y_{i}\right)$ é representado na malha cartesiana por $(i, j)$ e os pontos adjacentes são representados por $(i \pm 1, j \pm 1)$.

Para condição de regime transiente com propriedades constantes e sem geração interna de energia, a forma adequada da equação do calor é:

$$
\frac{1}{\alpha} \cdot \frac{\partial T}{\partial t}=\frac{\partial^{2} T}{\partial x^{2}}+\frac{\partial^{2} T}{\partial y^{2}}
$$

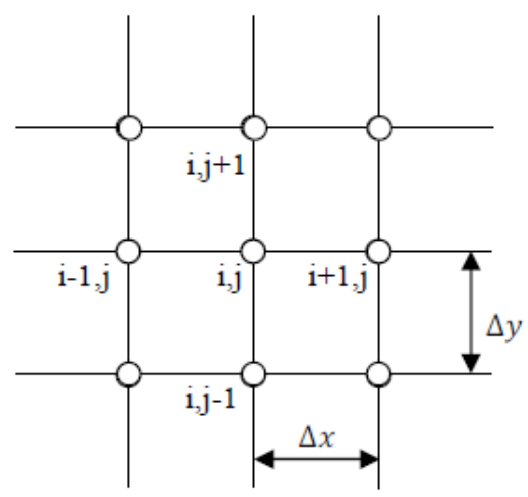

Figura 1 - Malha bidimensional. (MELO, 2011)
A fim de obter essa equação na forma de diferenças finitas, utilizam-se as aproximações por diferença central para as derivadas espaciais:

$\frac{\partial^{2} T}{\partial x^{2}} \approx \frac{T_{m+1, n}+T_{m-1, n}-2 T_{m, n}}{\Delta x^{2}}$

$\frac{\partial^{2} T}{\partial y^{2}} \approx \frac{T_{m, n+1}+T_{m, n-1}-2 T_{m, n}}{\Delta y^{2}}$

em que $\mathrm{m}$ e $\mathrm{n}$ fornecem as posições dos pontos nodais em relação aos eixos x e y, respectivamente. O número inteiro p é então introduzido com o intuito de discretizar a equação no tempo:

$$
t=p \Delta t
$$

Com isso, a expressão de diferenças finitas para a derivada em relação ao tempo torna-se igual a:

$$
\frac{\partial T}{\partial t} \approx \frac{T_{m, n}^{p+1}-T_{m, n}^{p}}{\Delta t}
$$

Assim, ao substituir as Equações 11, 12 e 14 na Equação 10, obtém-se a forma explícita da equação de diferenças finitas para o nó interior $\mathrm{m}, \mathrm{n}$ :

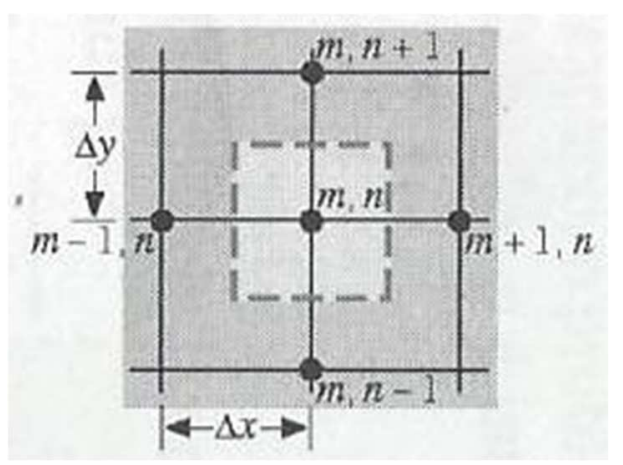

Figura 2 - Ponto nodal interior. (INCROPERA et al., 2008)

$$
\begin{aligned}
& \frac{1}{\alpha} \frac{T_{m, n}^{p+1}-T_{m, n}^{p}}{\Delta t} \\
& =\frac{T_{m+1, n}^{p}+T_{m-1, n}^{p}-2 T_{m, n}^{p}}{\Delta x^{2} p} \\
& +\frac{T_{m, n+1}^{p}+T_{m, n-1}^{p}-2 T_{m, n}^{p}}{\Delta y^{2}}
\end{aligned}
$$


Critério de estabilidade:

No método explícito as temperaturas são conhecidas no instante de tempo anterior (p) e, desse modo, a Equação 15 é uma aproximação por diferença adiantada para a derivada em relação ao tempo. Uma vez conhecidas as temperaturas nodais em $\mathrm{t}=0$, os cálculos são feitos para instantes de tempo sucessivos $(\mathrm{p}+1)$ separados por um intervalo de tempo $\Delta t$, e assim é obtida a distribuição de temperaturas transiente.

Ao explicitar a temperatura no novo instante de tempo e considerando que $\Delta x=\Delta y$, tem-se que:

$$
\begin{array}{r}
T_{m, n}^{p+1}=F o\left(T_{m+1, n}^{p}+T_{m-1, n}^{p}+T_{m, n+1}^{p}\right. \\
\left.+T_{m, n-1}{ }^{p}\right)+(1-4 F o) T_{m, n}^{p}
\end{array}
$$

Onde Fo é o número de Fourier em forma de diferenças finitas:

$$
F o=\frac{\alpha \Delta t}{\Delta x^{2}}
$$

Para evitar erros relacionados a oscilações do método, é adotado um critério de estabilidade, o qual é determinado com a exigência de que o coeficiente referente ao nó no instante anterior seja maior ou igual a zero. Dessa forma, é obtido o valor máximo do número de Fourier e, consequentemente, de $\Delta t$, que podem ser utilizados nos cálculos. Assim, para ponto nodal interior tem-se o seguinte critério de estabilidade:

$$
\text { Fo } \leq \frac{1}{4}
$$

Ressalta-se que as equações para demais configurações podem ser obtidas de modo análogo ao adotado para o ponto nodal interior ou ser deduzidas por meio de balanço de energia ao redor do nó, adotando-se a consideração de que todos os fluxos de calor sejam direcionados para o interior do mesmo.

$$
\begin{aligned}
& \text { (Taxa de energia que entra }) \\
& +(\text { Taxa de energia gerada }) \\
& =(\text { Taxa de energia acumulada })
\end{aligned}
$$

Assim sendo, a expressão do método explícito para ponto nodal em superfície plana com convecção, Figura 3, é definida por:

$$
\begin{aligned}
T^{p+1}{ }_{m, n}=F o( & 2 T^{p}{ }_{m-1, n}+T^{p}{ }_{m, n+1}+T^{p}{ }_{m, n-1} \\
& \left.+2 B i T_{\infty}\right) \\
& +(1-4 F o-2 B i F o) T^{p}{ }_{m, n}
\end{aligned}
$$

$$
F o(2+B i) \leq \frac{1}{2}
$$

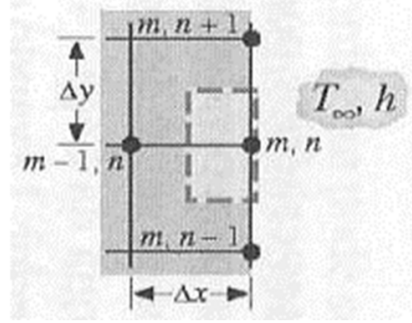

Figura 3 - Ponto nodal em uma superfície plana com convecção. (INCROPERA et al., 2008)

Para ponto nodal em vértice externo com convecção, Figura 4, por sua vez, a equação é a seguinte:

$$
\begin{array}{r}
T_{m, n}^{p+1}=2 F o\left(T^{p}{ }_{m-1, n}+T^{p}{ }_{m, n-1}+2 B i T_{\infty}\right) \\
+\left(1-4 F_{0}-4 B i F o\right) T^{p}{ }_{m, n}
\end{array}
$$

Critério de estabilidade:

$$
F o(1+B i) \leq \frac{1}{4}
$$

onde Bi é definido por:

$$
B i=\frac{h \Delta x}{k}
$$

Figura 4 - Ponto nodal em um vértice externo com convecção. (INCROPERA et al., 2008)

Existe também o método de diferenças finitas implícito, considerado uma aproximação por diferenças finitas atrasada da derivada em relação ao tempo. Como exemplo, tem-se a equação seguinte, deduzida por meio de balanço de energia para um nó interior: 


$$
\begin{aligned}
T_{m, n}^{p}=(1+4 F o) & T_{m, n}^{p+1} \\
& -F o\left(T_{m+1, n}^{p+1}+T_{m-1, n}^{p+1}\right. \\
& \left.+T_{m, n+1}{ }^{p+1}+T_{m, n-1}^{p+1}\right)
\end{aligned}
$$

Esse método é aconselhável para os casos em que o critério de estabilidade do método explícito exige valores para $\Delta t$ muito pequenos e, consequentemente, um número elevado de intervalos de tempo necessários para obtenção da solução. Diante de tais situações, o método implícito torna-se mais favorável já que possibilita o uso de intervalos de tempo maiores e não apresenta restrições em $\Delta x$ e $\Delta t$ (INCROPERA et al., 2008).

\subsection{Método da capacitância global}

Trata-se de um método simples bastante empregado na resolução de problemas transientes de transferência de calor (INCROPERA et al., 2008). Para ser aplicado, considera-se que a temperatura do sólido seja uniforme no espaço em todo instante de tempo, de tal forma que todo calor recebido ou gerado é difundido instantaneamente pelo sólido. Sabe-se que para que isso ocorresse de fato a condutividade térmica do sólido deveria tender ao infinito, o que não é possível. Todavia uma razoável aproximação para essa condição é aplicada se a resistência aos fluxos de calor no sólido for muito menor que a resistência à transferência de calor entre o sólido e sua vizinhança (SILVA, 2002).

Ao fazer um balanço de energia global em um sólido sob processo de resfriamento, obtém-se:

$$
-h A_{S}\left(T-T_{\infty}\right)=\rho V c \frac{d T}{d t}
$$

onde V é o volume do sólido, $\left[\mathrm{m}^{3}\right]$, c é o calor específico, [ $\mathrm{Jkg}^{-}$ $\left.{ }^{1} \mathrm{~K}^{-1}\right], \rho$ é a massa específica, $\left[\mathrm{kgm}^{-3}\right]$, e $A_{\mathrm{s}}$ é a área superficial, $\left[\mathrm{m}^{2}\right]$.

Definindo a diferença de temperaturas por $\theta$ e integrando a expressão obtida, tem-se:

$\theta \equiv T-T_{\infty}$

$\frac{\rho V c}{h A_{S}} \ln \frac{\theta_{i}}{\theta}=t$

Por meio desta equação torna-se possível determinar o tempo necessário para o sólido adquirir uma determinada temperatura ou mesmo calcular a temperatura alcançada em um instante específico. Além disso, por meio dela é definida a constante de tempo térmica:

$$
\tau_{t}=\frac{1}{h A_{S}}(\rho V c)=R_{t} C_{t}
$$

em que $R_{t}$ é a resistência à transferência de calor por convecção e $C_{t}$ é a capacitância térmica global do sólido. Nota-se que o aumento de uma dessas variáveis acarreta uma variação de temperatura mais lenta no sólido.

Quanto à energia transferida $\mathrm{Q}$ até um referido tempo, esta pode ser obtida por meio da seguinte equação:

$$
Q=(\rho V c) \theta i\left(1-\exp \left(\frac{-t}{\tau_{t}}\right)\right)
$$

Para efeito de validação do método, define-se o número de Biot:

$$
B i \equiv \frac{\left(\frac{L_{C}}{k A}\right)}{\left(\frac{1}{h A}\right)}=\frac{R_{\text {condução }}}{R_{\text {convecção }}}=\frac{h L_{C}}{k}
$$

Assim, se o número de Biot for inferior a 0,1 a resistência à condução no interior do sólido é desprezível em relação à resistência à convecção ao redor deste, então o método da capacitância global pode ser aplicado (INCROPERA et al., 2008).

Ressalta-se ainda que ao substituir o comprimento característico, $\mathrm{L}_{\mathrm{c}}$, na Equação 28, esta pode ser expressa em função dos números de Biot e Fourier:

$\frac{\theta}{\theta_{i}}=\frac{T-T_{\infty}}{T_{i}-T_{\infty}}=\exp (-B i . F o)$

onde $F o$ é definido por:

$$
F_{O} \equiv \frac{\alpha t}{L c^{2}}
$$

\section{METODOLOGIA}

Inicialmente, cortou-se as beterrabas em cubos de arestas de $5 \mathrm{~cm}$, medidos por uma régua com incerteza de $\pm 0,05 \mathrm{~cm}$.

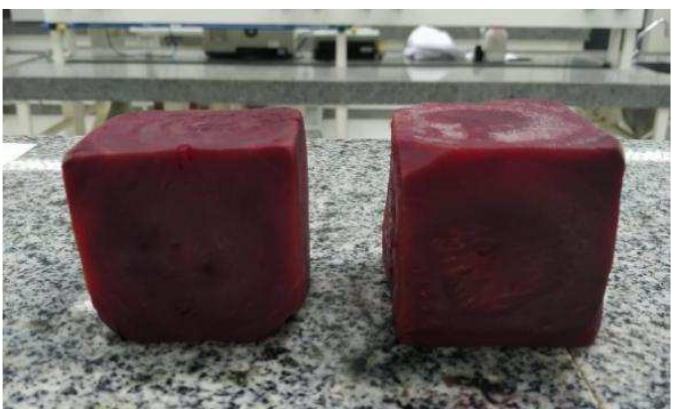

Figura 5 - Beterraba cortada em cubos. 
Simultaneamente, 1 L de água foi levada a ebulição dentro de um béquer com o auxílio de um ebulidor. A água foi transferida para uma panela de alumínio e o cubo de beterraba foi adicionado, permanecendo completamente submerso por 20 minutos com incerteza de medição de $\pm 2.10^{-4}$. Durante esse período, o aquecimento foi mantido a aproximadamente $300{ }^{\circ} \mathrm{C}$ em uma chapa de aquecimento. Os equipamentos foram montados conforme a imagem a seguir:

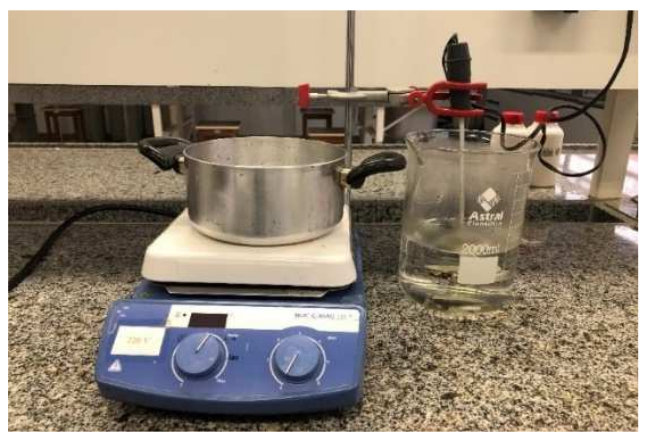

Figura 6 - Equipamentos utilizados para aquecimento.

Em seguida, o cubo foi retirado da panela e colocado sobre uma placa de poliestireno expandido. A partir de então, mediu-se, simultaneamente a temperatura dos pontos correspondentes nas faces frontal e traseira, como mostrado na Figura 7. A escolha de faces opostas tem como base comprovar empiricamente se a transferência de calor pode ser considerada bidimensional. Os pontos distam $2,15 \mathrm{~cm}$ entre si, tanto na horizontal, quanto na vertical e as medições de temperatura foram realizadas de 5 em 5 minutos até o tempo de 10 minutos.

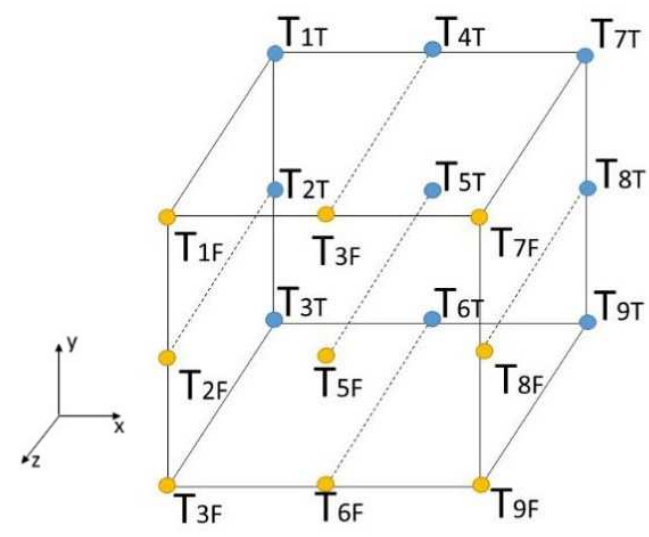

Figura 7 - Sistema de pontos de medição de temperatura na beterraba.
Os termômetros utilizados eram do tipo espeto digital das marcas "minipa" e "TP300" com incerteza de medição de $\pm 0,1^{\circ} \mathrm{C}$. O experimento foi realizado em duplicata.

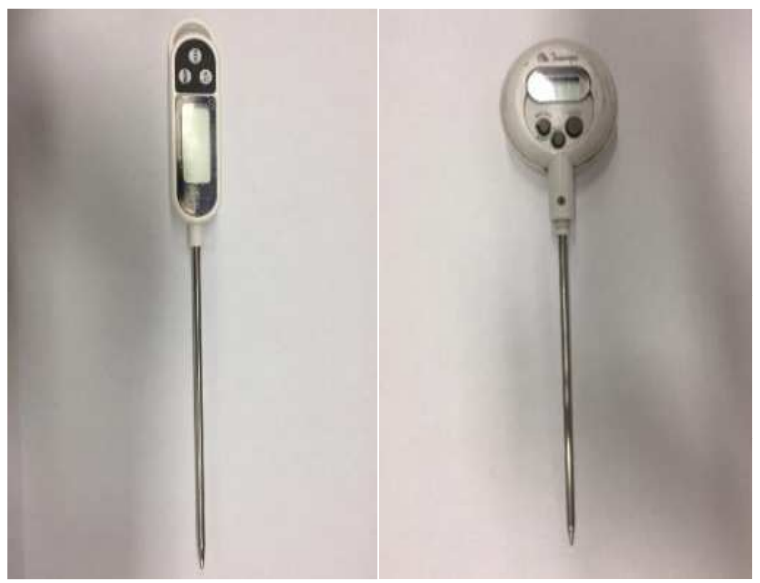

Figura 8 - Termômetros de espeto utilizados no experimento

\section{RESULTADOS}

As medidas de temperatura foram realizadas, inicialmente, em duas beterrabas, todavia, a primeira forneceu dados insatisfatórios devido, principalmente, ao fato de os termômetros utilizados serem diferentes e, consequentemente, possuírem tempos de resposta distintos. Além disso, a profundidade de inserção dos sensores e os respectivos pontos escolhidos para medição na beterraba em questão corroboraram para a obtenção de um perfil de temperaturas muito diferente do esperado. Dessa forma, levou-se em consideração apenas os dados referentes à segunda repetição, na qual as técnicas de aferição já haviam sido aprimoradas.

De posse das temperaturas nas faces frontal e traseira do alimento ao longo do tempo, Figura 7, observou-se que estas eram diferentes para pontos correspondentes, como mostrado na Tabela 1. A justificativa para esse fato está ligada, novamente, à diferença dos termômetros utilizados. Dessa forma, para a obtenção de dados mais próximos dos reais, a transferência de calor deveria ser considerada como tridimensional.

Apesar disso, como se trata de um experimento com fins prioritariamente didáticos, optou-se por considerar o sistema como sendo bidimensional e, dessa forma, tornar mais viável o tratamento dos dados. Essa simplificação foi realizada tirando-se a média das temperaturas dos pontos correspondentes em cada tempo, dando origem ao arranjo exibido a seguir. 


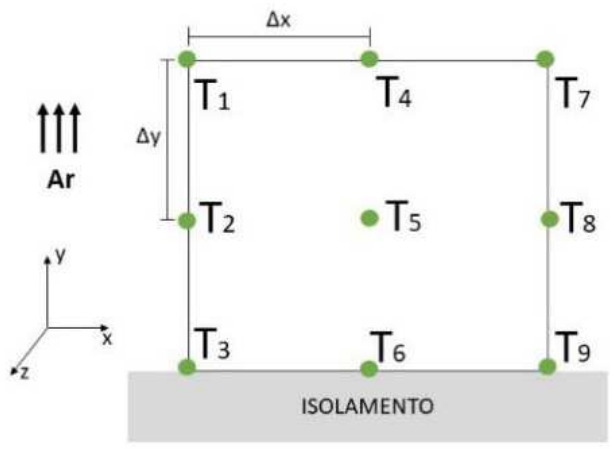

Figura 9 - Malha bidimensional resultante.

As temperaturas médias para os novos pontos nos tempos analisados encontram-se dispostos na Tabela 1, com suas respectivas incertezas (ANDRADE et al., 2017).
A partir dos dados experimentais obtidos no tempo 0, calculou-se as temperaturas nos pontos 1 a 9, em tempos futuros pela técnica de Diferenças Finitas - método explícito. Os possíveis intervalos de tempo a serem utilizados foram determinados isolando $F o$, e, posterirormente, $\Delta t$ nas equações de critério de estabilidade (Equações 18, 21 e 23). Os valores obtidos estão dispostos na Tabela 2.

$\mathrm{O} \Delta t$ escolhido deve ser menor ou igual àqueles calculados e, além disso, para fins práticos, um divisor exato do intervalo de tempo utilizado no experimento (300 s). Frente a isso, o $\Delta t$ escolhido foi de $150 \mathrm{~s}$. Para o cálculo das temperaturas por diferenças finitas foi necessária, ainda, a determinação de $\Delta \mathrm{x}$ e $\Delta \mathrm{y}$, feita durante o procedimento experimental. Deste modo, $\Delta \mathrm{x}$ e $\Delta \mathrm{y}$ foram estabelecidos arbitrariamente com valor igual a $0,0215 \mathrm{~m}$, de modo que os pontos coincidissem exatamente com aqueles mostrados na Figura 9. A temperatura do fluido $\left(T_{\infty}\right)$ manteve-se constante e

Tabela 1 - Temperaturas médias para os pontos correspondentes.

\begin{tabular}{cccccccccc}
\hline \multicolumn{8}{c}{ Temperatura/K } \\
\hline Tempo/s & $\mathbf{T}_{\mathbf{1}}$ & $\mathbf{T}_{\mathbf{2}}$ & $\mathbf{T}_{\mathbf{3}}$ & $\mathbf{T}_{\mathbf{4}}$ & $\mathbf{T}_{\mathbf{5}}$ & $\mathbf{T}_{\mathbf{6}}$ & $\mathbf{T}_{\mathbf{7}}$ & $\mathbf{T}_{\mathbf{8}}$ & $\mathbf{T}_{\mathbf{9}}$ \\
$\mathbf{0}$ & $329,8 \pm 0,1$ & $335,4 \pm 0,1$ & $340,0 \pm 0,1$ & $342,1 \pm 0,1$ & $346,5 \pm 0,1$ & $353,3 \pm 0,1$ & $326,1 \pm 0,1$ & $334,5 \pm 0,1$ & $339,4 \pm 0,1$ \\
$\mathbf{3 0 0}$ & $321,9 \pm 0,1$ & $326,2 \pm 0,1$ & $327,9 \pm 0,1$ & $330,0 \pm 0,1$ & $337,6 \pm 0,1$ & $341,9 \pm 0,1$ & $318,5 \pm 0,1$ & $325,0 \pm 0,1$ & $328,9 \pm 0,1$ \\
$\mathbf{6 0 0}$ & $316,2 \pm 0,1$ & $319,9 \pm 0,1$ & $320,6 \pm 0,1$ & $322,7 \pm 0,1$ & $329,4 \pm 0,1$ & $332,0 \pm 0,1$ & $313,1 \pm 0,1$ & $318,7 \pm 0,1$ & $321,3 \pm 0,1$
\end{tabular}

igual a $298,15 \mathrm{~K}$, sendo este o valor adotado para os cálculos subsequentes. Considerou-se, ainda, radiação negligenciável e não geração de energia para este experimento, de modo a simplificar o tratamento dos dados em análise.

Tabela 2 - Intervalos de tempo calculados a partir dos critérios de estabilidade.

\begin{tabular}{cc}
\hline Pontos & $\Delta t / \mathbf{s}$ \\
\hline 1 e 7 & 194 \\
$2,3,8$ e 9 & 224 \\
4 & 224 \\
5 e 6 & 265 \\
\hline
\end{tabular}

Para o cálculo do coeficiente de transferência de calor por convecção, fez-se necessário, anteriormente, o cálculo das difusividades térmicas da beterraba $(\alpha)$ e do ar $\left(\alpha_{a r}\right)$ e dos números de Prandtl $(\mathrm{Pr})$, Rayleigh $(\mathrm{Ra})$, Nusselt $(\mathrm{Nu})$ e Fourier
$(F o)$, os quais dependem das seguintes constantes termofísicas retiradas da literatura:

Tabela 3 - Constantes termofísicas obtidas para a beterraba na literatura.

\begin{tabular}{ccc}
\hline Constante & Valor & Referência \\
\hline $\boldsymbol{\rho} /\left(\mathrm{kgm}^{-3}\right)$ & 1530 & SWEAT (1974) \\
$\boldsymbol{C} \boldsymbol{p}\left(\mathrm{J} \mathrm{kg}^{-1} \mathrm{~K}^{-1}\right)$ & 900 & ASHRAE (1981) \\
$\boldsymbol{k}\left(\mathrm{W} \mathrm{m}^{-1} \mathrm{~K}^{-1}\right)$ & $6,01.10^{-1}$ & SWEAT (1974) \\
\hline
\end{tabular}

Tabela 4 - Constantes termofísicas obtidas para o ar na literatura.

\begin{tabular}{ccc}
\hline Constante & Valor & Referência \\
\hline $\boldsymbol{\mu}_{\boldsymbol{a r}}\left(\mathrm{kg} \mathrm{m}^{-1} \mathrm{~s}^{-1}\right)$ & $1,84.10^{-5}$ & FOX e McDONALD (2010) \\
$\boldsymbol{\rho}_{\boldsymbol{a r}}\left(\mathrm{kg} \mathrm{m}^{-3}\right)$ & 1,19 & FOX e McDONALD (2010) \\
$\boldsymbol{k}_{\boldsymbol{a r}}\left(\mathrm{W} \mathrm{m}^{-1} \mathrm{~K}^{-1}\right)$ & $2,63.10^{-2}$ & PERRY e GREEN (1997) \\
$\boldsymbol{C} \boldsymbol{p}_{\boldsymbol{a r}}\left(\mathrm{J} \mathrm{kg}^{-1} \mathrm{~K}^{-1}\right)$ & 1007 & PERRY e GREEN (1997) \\
\hline
\end{tabular}


Os valores obtidos foram, portanto, $4,36.10^{-7} \mathrm{~m}^{2} \mathrm{~s}^{-1}$ para $\alpha, 2,19.10^{-5} \mathrm{~m}^{2} \mathrm{~s}^{-1}$ para $\alpha_{\text {ar }}, F o$ igual a $(0,1416 \pm 0,0066), \operatorname{Pr}$ com valor de 0,7045 e $R a$ igual a $(10218,1907 \pm 28,0400)$, incluindose os valores das incertezas propagadas, conforme discutido e apresentado em Andrade et al. (2017).

Devido à geometria escolhida, foi preciso calcular o coeficiente de transferência de calor por convecção do ar em contato com placas verticais e placas horizontais. Para isso, utilizando as devidas correlações estabelecidas para a temperatura ambiente, $298,15 \mathrm{~K}$, como mostrado no tópico 2.2 da revisão bibliográfica, foram calculados dois valores para $N u$, obtendo-se assim, $(5,4540 \pm 0,0032)$ para a placa vertical e $(5,4292 \pm 0,0037)$ para a placa horizontal. Estes, substituídos na Equação 6, fornecem os valores de $(10,1851 \pm 0,0006) \mathrm{Wm}^{-2} \mathrm{~K}^{-1}$ e $(10,1388 \pm 0,0007) \mathrm{Wm}^{-2} \mathrm{~K}^{-1}$ para os coeficientes de transferência de calor por convecção em placas verticais e horizontais, respectivamente. De posse desses dados, foi possível calcular Biot e, dessa forma, aplicar, de fato, as equações de diferenças finitas para cada nó.

Para o nodo interno designado pelo ponto $\mathrm{T}_{5}$ na Figura 9, utilizou-se a Equação 16 para cálculo das temperaturas nos tempos futuros. $\mathrm{O}$ mesmo foi efetuado para o ponto $\mathrm{T}_{6}$, considerado como ponto interno devido ao plano de simetria formado pelo isolamento da superfície inferior da beterraba. Para os pontos $T_{2}, T_{3}, T_{4}, T_{8}$ e $T_{9}$, os quais estão em uma superfície plana com convecção, empregou-se a Equação 20. Os pontos 3 e 9 foram assim considerados devido, novamente, à simetria. Aplicou-se, por fim, a Equação 22 para os pontos nodais $\mathrm{T}_{1}$ e $\mathrm{T}_{7}$, vértices externos com convecção. Os resultados obtidos estão dispostos na Tabela 5. Observados a propagação de incertezas, conforme discutido e apresentado em Andrade et al. (2017).
De posse dos dados experimentais e calculados para cada intervalo de tempo averiguado, foi possível compará-los através do gráfico da Figura 10, plotado com auxílio do software OriginPro 8.

Ao analisar as curvas correspondentes ao método numérico, observa-se que estas apresentam uma queda de temperatura mais suave quando comparadas com os pontos obtidos experimentalmente. Esse comportamento é observado para todos os pontos de coleta, sendo que, para os pontos $\mathrm{T}_{5} \mathrm{e}$ $\mathrm{T}_{6}$, que são nodos internos, verifica-se que a diferença entre os dois métodos foi menos significativa.

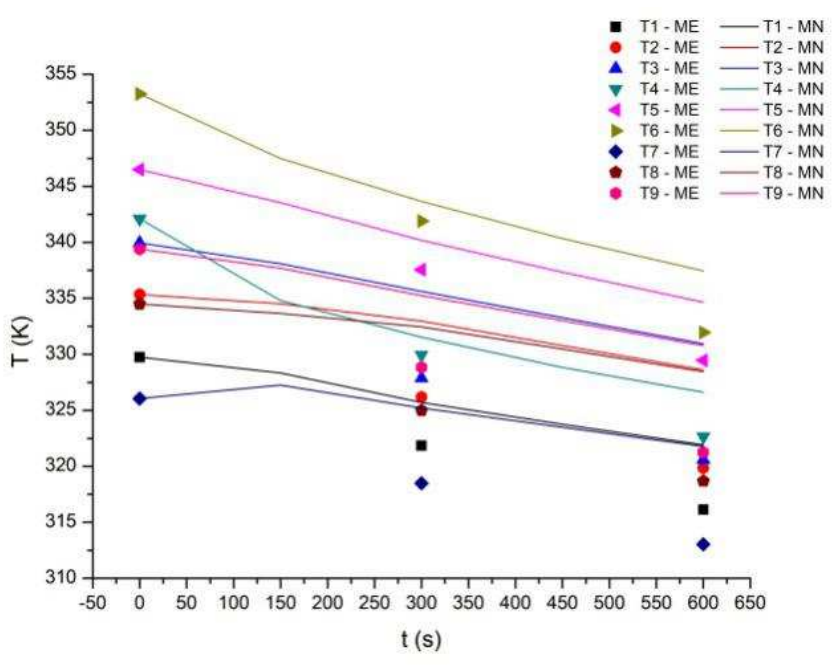

Figura 10 - Variação da temperatura de acordo com os dados experimentais (ME) e calculados (MN) pelo método numérico de diferenças finitas.

Tabela 5 - Temperaturas obtidas a partir da técnica de diferenças finitas.

\begin{tabular}{|c|c|c|c|c|c|c|c|c|c|}
\hline \multicolumn{10}{|c|}{ Temperatura/K } \\
\hline Tempo/s & $\mathbf{T}_{1}$ & $T_{2}$ & $T_{3}$ & $T_{4}$ & $\mathbf{T}_{5}$ & $T_{6}$ & $T_{7}$ & $T_{8}$ & $T_{9}$ \\
\hline 0 & $329,8 \pm 0,1$ & $335,4 \pm 0,1$ & $340,0 \pm 0,1$ & $342,1 \pm 0,1$ & $346,5 \pm 0,1$ & $353,3 \pm 0,1$ & $326,0 \pm 0,1$ & $334,5 \pm 0,1$ & $339,4 \pm 0,1$ \\
\hline 150 & $328,3 \pm 0,2$ & $334,5 \pm 0,1$ & $338,1 \pm 0,1$ & $334,8 \pm 0,4$ & $343,6 \pm 0,1$ & $347,5 \pm 0,3$ & $327,3 \pm 0,2$ & $333,6 \pm 0,1$ & $337,7 \pm 0,1$ \\
\hline 300 & $325,7 \pm 0,3$ & $333,0 \pm 0,4$ & $335,6 \pm 0,3$ & $331,5 \pm 0,4$ & $340,2 \pm 0,3$ & $343,7 \pm 0,4$ & $325,2 \pm 0,3$ & $332,4 \pm 0,2$ & $335,2 \pm 0,3$ \\
\hline 600 & $321,9 \pm 0,5$ & $328,6 \pm 0,5$ & $330,9 \pm 0,5$ & $326,6 \pm 0,5$ & $334,7 \pm 0,5$ & $337,4 \pm 0,6$ & $321,8 \pm 0,4$ & $328,4 \pm 0,4$ & $330,8 \pm 0,5$ \\
\hline
\end{tabular}

Como o cálculo do coeficiente de transferência de calor por convecção é um procedimento que envolve inúmeros passos, correlações e considerações, os valores encontrados para esses parâmetros podem carregar erros inerentes ao processo de cálculo. Dessa forma uma possível justificativa para os menores erros observados nos pontos $\mathrm{T}_{5}$ e $\mathrm{T}_{6}$ é o fato de nodos internos 
não estarem diretamente expostos à convecção. Ressalta-se ainda, que, em um trabalho realizado por Zanini (2009), estudou-se a aplicação de uma modelagem utilizando diferenças finitas na otimização do processamento térmico de alimentos enlatados. Os resultados obtidos demonstraram que, para pontos localizados na superfície dos alimentos analisados, o erro associado ao procedimento é relativamente maior do que aquele encontrado para pontos internos, o que também foi observado no presente estudo.

Além disso, ao analisar a variação de temperatura nos pontos $\mathrm{T}_{2}$ e $\mathrm{T}_{8}$ nota-se que, tanto os pontos experimentais quanto as curvas obtidas via método numérico para os pontos em questão encontram-se muito próximas entre si durante todo o período de análise. Esse comportamento já era de se esperar, visto que, pela geometria escolhida nesse experimento, o plano yz que corta os pontos $\mathrm{T}_{4}, \mathrm{~T}_{5}$ e $\mathrm{T}_{6}$ representa um plano de simetria. Essa tendência também é observada nos outros pontos que são simétricos em relação ao plano citado, o que confirma a simetria. São eles: $\mathrm{T}_{2}$ e $\mathrm{T}_{8} ; \mathrm{T}_{1}$ e $\mathrm{T}_{7}$.

Uma tendência que destoa das demais curvas geradas pelo método de diferenças finitas pode ser observada para os pontos $\mathrm{T}_{4} \mathrm{e} \mathrm{T}_{7}$. $\mathrm{O}$ primeiro deles apresenta uma queda brusca de temperatura no início do período de análise, o que se deve, provavelmente, ao fato de este ser o único ponto sujeito à troca de calor por convecção em superfície horizontal. Os demais são vértices externos, sujeitos à troca de calor por convecção tanto na horizontal quanto na vertical, pontos de superfície vertical e pontos internos.

Já na curva $\mathrm{T}_{7}$ nota-se um comportamento crescente até os 150 segundos, o que não é esperado para um objeto aquecido exposto à um fluido em menor temperatura. Mais uma vez, o principal responsável pelo ocorrido é, provavelmente, o tempo decorrido até a medição da temperatura do ponto em questão, visto que este foi o último dado tomado no tempo 0. Medições mais ágeis confeririam a este uma maior temperatura inicial e um comportamento exclusivamente decrescente da curva.

Com o intuito de aplicar o método da Capacitância Global para o cálculo da variação de temperatura da beterraba, é necessário considerar a temperatura do alimento uniforme para cada instante de tempo, e, para isso, utilizou-se a média dos valores nos nove pontos da superfície.

A partir do valor médio encontrado no tempo 0 , realizou-se o cálculo para tempos futuros, com intervalos de tempo de $150 \mathrm{~s}$, finalizando em $600 \mathrm{~s}$. Para a realização deste procedimento numérico, utilizaram-se os valores de coeficiente de transferência de calor por convecção, $h$, e difusividade térmica da beterraba, $\alpha$. A Tabela 6 sumariza estes dados experimentais e os respectivos erros associados, para efeito de comparação.
Tabela 6 - Temperaturas calculadas pelo método da capacitância global.

\begin{tabular}{|c|c|c|c|}
\hline \multicolumn{4}{|c|}{ Temperatura/K } \\
\hline Tempo/s & Experimental & Capacitância Global & $\begin{array}{c}\text { Erro } \\
\text { (\%) }\end{array}$ \\
\hline 0 & $338,5 \pm 0,1$ & $338,5 \pm 0,0$ & 0 \\
\hline 150 & - & $337,7 \pm 0,0$ & - \\
\hline 300 & $328,6 \pm 0,1$ & $336,9 \pm 0,1$ & 2,5 \\
\hline 450 & - & $336,1 \pm 0,1$ & - \\
\hline 600 & $321,5 \pm 0,1$ & $335,3 \pm 0,1$ & 4,3 \\
\hline
\end{tabular}

De acordo com Incropera et al. (2008), para que o método da capacitância global seja válido, é necessário que o valor encontrado para Biot seja menor que 0,1. Como evidenciado anteriormente, ambos os valores encontrados para esse parâmetro, referentes à troca de calor por convecção tanto em placas horizontais como verticais, foram maiores do que este limite. Além disso, ainda segundo Incropera et al. (2008), o método em questão se aplica somente quando a temperatura do corpo em análise pode ser considerada uniforme e quando este está completamente envolto pelo fluido responsável pela troca de calor por convecção.

Por outro lado, no presente experimento o corpo estava isolado em uma de suas faces e foi mostrado que a temperatura varia substancialmente de ponto a ponto na beterraba em um mesmo instante de tempo. Sendo assim, não era esperado que os resultados obtidos através dessa metodologia numérica fossem próximos aos dados experimentais. Apesar da simplicidade associada a este método, o seu uso por diversas vezes se torna restrito, e os valores obtidos pelo procedimento podem diferir dos dados experimentais.

Quando se trata do tempo máximo de exposição do alimento ao ar para evitar a contaminação microbiológica, observa-se que, ainda no tempo 0 , já existiam pontos com temperatura inferior a 333 K. Segundo Ball e Olson (1957), esses pontos já estariam susceptíveis a contaminação, o que poderia oferecer riscos ao consumidor. Dentre as justificativas para essa ocorrência, destaca-se o elevado tempo requerido para a aferição das temperaturas em todos os 9 pontos da face. $\mathrm{O}$ período decorrido entre a primeira e a última medição naquele que foi considerado o tempo 0 , contribuiu para que os últimos pontos a serem avaliados já houvessem resfriado consideravelmente. Além disso, como se trata de um experimento com fins didáticos, não foi levado em consideração o ponto de cozimento do alimento, de forma que, um maior tempo em contato com a água em ebulição, poderia conferir maiores temperaturas iniciais e, consequentemente, um maior tempo de exposição ao ar permitido. 


\section{CONSIDERAÇÕES FINAIS}

A aplicação do método de diferenças finitas explícito e capacitância global permitiu a análise da variação de temperatura nas faces da beterraba nos intervalos de 150 segundos a fim de estabelecer o tempo que o alimento poderia estar exposto ao ambiente sem risco de contaminação.

Para utilizar os métodos, foi necessário realizar algumas considerações que além de simplificar os cálculos, também atribuíram para o aumento de alguns erros nos resultados obtidos. Dentre todos os equipamentos utilizados o item que mais contribuiu para a incerteza foram os termômetros. Diante disso, é valido afirmar que equipamentos com uma precisão maior poderiam melhorar os resultados obtidos. Por outro lado, durante o experimento a falta de uma grande quantidade de termômetros tornou inviável a medição de todos os pontos simultaneamente. Essa limitação impediu a aferição de todas as temperaturas de uma face em um mesmo instante, aumentando a parcela de erros.

Ao aplicar o método de capacitância global, verificou-se que o Biot encontrado não está na faixa de valores adequados a aplicação. Dessa forma, pode-se considerar que a aplicação desse método embora estabeleça uma estimativa, não se trata de um valor próximo da realidade.

Tendo em vista as circunstâncias nas quais esse trabalho foi produzido, pode-se considerar que os resultados obtidos foram razoáveis, dados os materiais que estavam disponíveis para a realização da análise.

$\mathrm{Na}$ avaliação dos dados obtidos, verificou-se que, antes mesmo da análise em tempos futuros, dois dos pontos superficiais do alimento já haviam alcançado as temperaturas de 52,9 e $56,6^{\circ} \mathrm{C}$, ou seja, ultrapassado a temperatura de $60{ }^{\circ} \mathrm{C}$, considerada como limite. Assim, é valido ressaltar a importância do cozimento de alimentos por tempo considerável, e atentar-se para o quão rápido ocorre o resfriamento destes.

\section{AGRADECIMENTOS}

Os autores agradecem o apoio por parte da Universidade Federal de Viçosa (UFV). O presente trabalho foi realizado com apoio da Coordenação de Aperfeiçoamento de Pessoal de Nível Superior - Brasil (CAPES) - Código de Financiamento 001 e da Fundação de Amparo à Pesquisa do Estado de Minas Gerais (FAPEMIG).

\section{R E F E R E N C I A S}

ABIA - ASSOCIAÇÃO BRASILEIRA DE INDUSTRIA DE ALIMENTOS. Indústria de Alimentos fecha 2018 com aumento de 2,08\% em faturamento. São Paulo, 2019. Disponível em: <https://www.abia. org.br/vsn/tmp_2.aspx?id=393>. Acesso em: 12 nov. 2019.
AMÊNDOLA, M.; TUREL, B.; Uso de um esquema implícito e de splines para a simulação numérica do processo de resfriamento de frutas esféricas. Revista brasileira de Engenharia Agrícola e Ambiental, v.9, n.1, p78-82, 2005.

AMERICAN SOCIETY OF HEATING, REFRIGERATING AND AIR-CONDITIONING ENGINEERS (ASHRAE). Ashrae Handbook, 1981 Fundamentals. Atlanta, GA: ASHRAE Inc., 1981.

AMORIM, H. C. Modelagem e Simulação da Transferência de Calor em Alimentos com Forma Esferoidal Prolata. Estudo de Caso: Resfriamento e Congelamento de Banana. Dissertação de mestrado. Universidade Federal de Campina Grande, PB, 2016.

ANDRADE, A. C. A.; REIS, H. F. A. F.; SIQUEIRA, A. M. de O.; MADUREIRA, M. F.; GOUVEA, N. A.; GONZAGA, L. F.; MENEZES, T. L. de; FREIRE, B. H. de F. PROPAGAÇÃO DE INCERTEZAS: UM EXPERIMENTO ACADÊMICO SIMPLES. The Journal of Engineering and Exact Sciences, [S. 1.], v. 3, n. 3, p. 358-368, 2017. DOI: 10.18540/jcecvl3iss3pp358$368 . \quad$ Disponível em: https://periodicos.ufv.br/jcec/article/view/24469416030 32017358. Acesso em: 19 jan. 2020.

ANVISA. Vigilância epidemiológica das doenças transmitidas por alimentos no Brasil, 1999-2004. Boletim eletrônico epidemiológico. Brasília, DF, ano 5, n 6, p. 1-7, dez. 2005. Disponível em <http://www.saude.gov .br/boletins-epidemiologicos $>$. Acesso em: 12, nov. 2019.

BALL, C. O.; OLSON, F. C. W. Sterilization in food technology: theory, practice, and calculations. New York: MacGraw-Hill, 1957, 645p.

ÇENGEL, Y. A.; GHAJAR, A. J. Transferência de Calor e Massa: Uma abordagem prática. 4st ed., Porto Alegre: McGraw-Hill, 2012.

DA ROSA, C. T. W.; DA ROSA, A. B.; TRENTIN, M. A.; GIACOMELLI, A. C. Experimento de condução térmica com e sem uso de sensores e Arduino. Caderno Brasileiro de Ensino de Física, Florianópolis, v. 33, n. 1, p. 292305, abr. 2016.

FOX, R. W.; McDONALD, A. T. Introdução à mecânica dos fluidos. Tradução de Ricardo Nicolau Nassar Koury,Luiz Machado. 7. ed. Rio de Janeiro: LTC, 2010. 710 p. Inclui índice. ISBN 85-216-1757-0.

GARCIA, R. L.; AMARAL, R. A.; ZABADAL, J.; PIBERNAT, C. C.; JUCHEM, F.; SCHMITZ, A. Resfriamento de um cilindro de aço: estudo experimental da convecção e radiação do calor. Rev. Bras. Ensino Fís., São Paulo, v. 39, n. 4, e4501, 2017.

INCROPERA, F. P.; DEWITT, D. P.; BERGMAN, T. L.; LAVINE, A. S. Fundamentos de Transferência de calor e de massa. 6st ed., Rio de Janeiro: LTC, 2008. 
MELO, K. J. M. Aplicação do método das diferenças finitas explícito na solução da equação do calor para o caso transiente e unidimensional. Monografia. Universidade Federal Rural do Semi-Árido. Angicos, RN, 2011.

PERRY, R. H.; GREEN, D. W. Perry's chemical engineering handbook. 7th ed. New York: McGraw-Hill, 1997. ISBN 0-07-049841-5.

REDE NACIONAL DE ESTAÇÕES GRAVIMÉTRICAS ABSOLUTAS - RENEGA. Relatório parcial sobre o valor da aceleração da gravidade local na Estação Viçosa. Departamento de Engenharia - Universidade Federal de Viçosa, Viçosa, MG, 1989.

RODRIGUES, L. J. Análise transiente da transferência de calor em um tubo através do método das diferenças finitas. 2011. 20 páginas. Monografia (Trabalho de Conclusão do Curso em Engenharia Mecânica) - Departamento de Engenharia Mecânica Universidade Federal do Rio Grande do Sul, Porto Alegre, 2011.

RUGGIERO, M. A. G.; LOPES, V. L. R. Cálculo numérico: aspectos teóricos e computacionais. 2st ed., São Paulo: Pearson Makron Books, 1996.

SILVA, G. C.; LOPES, R. C.; LOPES, A. P. O método dos elementos finitos em treliças planas na disciplina de mecânica computacional. Anais do Congresso Brasileiro de Ensino de Engenharia, 2007.

SILVA, J. B. Secagem de sólidos em camada fina via análise concentrada: Modelagem e simulação. Dissertação de mestrado. Universidade Federal da Paraíba. Campina Grande, PB, 2002.

SWEAT, V. E. Experimental values of thermal conductivity of selected fruits and vegetables. Journal of Food Science. Agricultural Engineering Dept., Purdue University, West Lafayette, v. 39, p 1080-1083, 1974. Disponível em: https://onlinelibrary.wiley.com/doi/abs/10.1111/j.13652621.1974.tb07323.x. Acesso em: 11 nov. 2019.

TERUEL, B.; CORTEZ, L. A.; LEAL, P.; LIMA, A. G. B. Estudo teórico do resfriamento com ar forçado de frutas de geometrias diferentes. Revista Ciência e Tecnologia de Alimentos, v.21, n.2, p.228-235, 2001.

ZANINI, K. R. P. Contribuição ao emprego do modelo da difusão na otimização do processamento térmico de alimentos enlatados. Dissertação de mestrado. Centro Universitário do Instituto Mauá de Tecnologia. São Caetano do Sul, SP, 2009. 